Published in final edited form as:

Curr Allergy Asthma Rep. 2013 August ; 13(4): 361-370. doi:10.1007/s11882-013-0350-3.

\title{
Autoimmunity in Immunodeficiency
}

\author{
Krista Todoric ${ }^{1}$, Jessica B. Koontz ${ }^{2}$, Daniel Mattox ${ }^{2}$, and Teresa K. Tarrant ${ }^{2}$ \\ ${ }^{1}$ Division of Allergy and Immunology, Dept of Pediatrics, University of North Carolina Hospitals, \\ Chapel Hill, NC 27599, USA \\ ${ }^{2}$ Thurston Arthritis Research Center, Divisions of Rheumatology \& Allergy and Immunology, Dept \\ of Medicine, University of North Carolina Hospitals, Chapel Hill, NC 27599, USA
}

\begin{abstract}
Primary immunodeficiencies (PID) comprise a diverse group of clinical disorders with varied genetic defects. Paradoxically, a substantial proportion of PID patients develop autoimmune phenomena in addition to having increased susceptibility to infections from their impaired immunity. Although much of our understanding comes from data gathered through experimental models, there are several well-characterized PID that have improved our knowledge of the pathways that drive autoimmunity. The goals of this review will be to discuss these immunodeficiencies and to review the literature with respect to the proposed mechanisms for autoimmunity within each put forth to date.
\end{abstract}

\section{Keywords}

Review; Autoimmunity; Primary immunodeficiency; Autoreactive; Autoantigen; Tolerance; Apoptosis

\section{INTRODUCTION}

Primary immunodeficiencies (PID) comprise a diverse group of more than 150 inherited and sporadic disorders. For some, an underlying genetic mutation has been identified, and for others, the defect is not yet known. While clinical characteristics vary, all PID lead to increased susceptibility to infection. Additionally, these patients somewhat paradoxically develop autoimmune phenomena more frequently than the general population [1].

Central tolerance drives the deletion of the majority autoreactive $\mathrm{T}$ and $\mathrm{B}$ cells. Even within healthy individuals, a few of these cells likely escape the thymus, but peripheral mechanisms normally prevent reactivity. In the same way that genetic defects may contribute to immunodeficiency and the abnormal development and maintenance of T and B cells, these defects may predispose PID patients to autoimmune phenomena. At this time, accepted theories of autoimmunity within PID center on failure of mechanisms of tolerance but also extend to include mechanisms of apoptosis or proliferative defects, signaling pathway or immune-mediated clearance defects, or aberrations in innate cellular mechanisms.

Corresponding Author: Teresa K. Tarrant, The University of North Carolina at Chapel Hill, CB\# 7280, 3330 Thurston Building, Chapel Hill, NC 27599-7280, USA, teresa_tarrant@med.unc.edu.

Disclosure Krista Todoric declares that she has no conflict of interest.

Jessica B. Koontz declares that she has no conflict of interest.

Daniel Mattox declares that he has no conflict of interest.

Teresa K. Tarrant has served as a consultant for Roche. 
Frequently, more than one mechanism may be responsible for the autoimmune manifestations present in a single PID.

Although much of our understanding comes from data gathered through experimental models, there are several well-characterized PID that have improved our knowledge of the pathways that drive autoimmunity. The goals of this review will be to discuss these immunodeficiencies and review the literature with respect to the proposed mechanisms for autoimmunity within each put forth to date (see Table 1 for a summary of these mechanisms).

\section{DEFECTS OF TOLERANCE}

\section{A. CENTRAL TOLERANCE}

Central tolerance is the deletion of autoreactive $\mathrm{T}$ cells that recognize self-peptides presented on thymic epithelium primarily under the direction of the autoimmune regulator (AIRE) gene. Thus, autoimmune mechanisms that may involve defects in central tolerance are best observed in the primary immunodeficiency autoimmune polyendocrinopathy, candidiasis, and ectodermal dystrophy (APECED) which results from a mutation in AIRE.

\section{AUTOIMMUNE POLYENDOCRINOPATHY, CANDIDIASIS AND ECTODERMAL} DYSPLASIA-Within the thymus, AIRE controls the expression of a wide array of selfpeptides found in peripheral organs, such as insulin, thyroid peroxidase, thyroglobulin, 21hydroxylase, and myelin basic protein, and it is responsible for the maintenance of thymic epithelial progenitor cells [2-4]. The zinc-finger domain of AIRE functions to mediate the transfer of ubiquitin to specific proteins, hence targeting them to a variety of pathways such as degradation [5-7]. Some peripheral self-peptides, such as C-reactive protein and glutamic-acid decarboxylase, are also expressed in the thymus but are not controlled by AIRE; the process of their expression is not well understood [4]. Although its major role and function are thought to be through regulation of central tolerance, AIRE is also found to a lesser degree in the spleen, pancreas, adrenal cortex, and lymph nodes, also suggesting a role in peripheral tolerance $[8,9]$.

Typical clinical presentation of APECED includes the triad of cutaneo-mucus candidiasis occurring by age 5 (in $75 \%$ ) followed by autoimmune-mediated hypoparathyroidism occurring by age 10 (in $89 \%$ ) and adrenocortical failure occurring by age 15 (in $60 \%$ ) [912]. Other autoimmune manifestations include hepatitis, primary biliary cirrhosis (PBC), thyroiditis, hypogonadism, autoimmune hemolytic anemia (AHA), pernicious anemia, type 1 diabetes mellitus (T1DM), vitiligo, alopecia, keratoconjunctivitis, and ectodermal dysplasia $[6,7,9,10,13]$.

Autoimmunity in APECED is felt to result from failure of deletion of autoreactive T cells as they undergo thymic selection. Evidence for this may be highlighted by the recent work of Su et al. who showed in a murine model that failure of thymic expression of myelin protein zero (controlled by AIRE) leads to CD4+ T cell infiltration in peripheral nerves, with subsequent IFN- $\gamma$ production and demyelination [14]. In this model, CD4+ T cells were sufficient to transfer disease and mediate this Th1 response against peripheral nerves [14].

Failure of central tolerance appears to be the primary driving force for these autoimmune manifestations, although further work on peripheral tolerance mechanisms may change our understanding of these manifestations in the future. Teh et al. have also explored the possibility of a second hit in peripheral tolerance that could lead to more rapid manifestation of autoimmunity in APECED models [15]. Their murine work shows that absence of AIRE coupled with absence of CBL-B, an inhibitor of signaling pathways (P13K and NFkB) 
normally activated by TCR-CD28 interaction in mature T cells, results in the rapid progression of pancreatic autoimmune disease [15]. This rapid disease is hypothesized to result from a combination of self-reactive $\mathrm{T}$ cells escaping from the thymus and the failure of these cells to undergo anergy due to lack of CBL-B [15]. Further, it has been suggested, in chronic cutaneo-mucus candidiasis patients with AIRE deficiency, that there is defective Th17 differentiation (due to STAT 1/3 mutations), receptor function, and cytokine production (IL17-F loss-of-function) as well as the development of autoantibodies against type 1 interferons and Th17 cytokines that are contributing to disease pathogenesis [16].

\section{B. PERIPHERAL TOLERANCE}

As implicated above, the absence of peripheral tolerance mechanisms that induce anergy in T cells through strength of TCR signaling may also contribute to the activation of autoreactive cells. While not well elicited, it is also believed that the innate immune system also contributes to peripheral tolerance. Diseases whereby one or some of these mechanisms are speculated to be at play include hyper IgM syndrome ( $\mathrm{HIMM})$, which results from a mutation of class switch genes, X-linked agammaglobulinemia (XLA) which results from a mutation in Bruton's tyrosine kinase (Btk), common variable emmunodeficiency (CVID) of varied genetic defects, and immunodysregulation, polyendocrinopathy, enteropathy, Xlinked (IPEX), which results from a defect in forkhead box protein P3 (FoxP3).

HYPER IGM SYNDROME-HIgM syndrome is a group of defects in class switch recombination that results in normal/high levels of $\operatorname{IgM}$, low levels of $\operatorname{IgA}$ and $\mathrm{IgG}$, and an inability to produce memory B cells $[8,10,17]$. There are seven identified molecular subsets: X-linked defect in CD40 ligand (type 1,70 \%), autosomal recessive/dominant defect in activation-induced cytidine deaminase (AID) (type $2,<1 \%$ ), autosomal recessive defect in CD40 (type 3,10\%), autosomal recessive defect in uracil-DNA-glycosylase (UDG) (type 5, $5 \%$ ), X-linked defect in nuclear factor kappa B essential modulator (NEMO) (type 6,1-2\%), and defect in IkBa (type 7); type 4 is an unknown defect [17-19].

Autoimmune manifestations most frequently occur in patients with AID (25\%), NEMO (23 $\%$ ), and CD40L ( $20 \%$ ) defects. These patients may develop AHA, IBD, and polyarteritis [8, 10, 20]. Additionally, those with AID and CD40L defects may develop thyroiditis and cytopenias (ITP in AID and neutropenia in CD40L), while those with AID defects have uniquely developed hepatitis, uveitis, and T1DM $[8,10,20]$.

The proteins described above are critical components in signaling and DNA recombination pathways that result in immunoglobulin class-switching and cellular activation. Normally, binding of CD40 (found on B cells and antigen-presenting cells) to transiently expressed CD40 ligand (CD40L) (found on activated T cells) induces a cytoplasmic domain to bind to TNF-receptor-associated-factor (TRAF) proteins, which in turn leads to further downstream transcription effects mediated by nuclear factor (NF)-kB. This signal results in B cell growth and differentiation, germinal center formation, and immunoglobulin class switching as well as elimination of autoreactive B cells in the bone marrow or periphery (as seen in mouse models) $[8,17,18,21]$. CD40-CD40L also provides a co-stimulatory signal for $\mathrm{T}$ cell activation and primes T lymphocytes for antigen-presentation [17-19]. T cell activation is also impacted by cytokine secretion from innate cells (such as antigen-presenting cells, platelets, endothelial cells, and endothelial cells) expressing CD40; hence, impaired functioning and maturation of these types of cells due to defects in CD40 may also impact $\mathrm{T}$ cell regulation $[17,19,22]$. In addition, a loss of CD40L signaling affects thymic epithelial cells, thus leading to a potential loss of development of $\mathrm{T}$ regulatory cells $[17,21,23]$. 
X-LINKED AGAMMAGLOBULINEMIA-X-linked agammaglobulinemia (XLA) is the result of a mutation in Bruton's tyrosine kinase (Btk), a signal transduction molecule needed in the process of B cell development, proliferation, and survival signaling [24]. These patients have few to no circulating B cells due to arrest of the B cell development at the proB stage [23, 25]. Clinical manifestations include bacterial sinopulmonary infection, eczema, and diarrhea, with diagnosis usually occurring within the first few years of life [9]. Autoimmune manifestations include AHA, arthritis, alopecia, scleroderma, dermatomyositis, inflammatory bowel disease (IBD), and T1DM [8, 9, 11, 25].

Mechanisms of autoimmunity may be similar to those of HIgM. Alternatively, abnormalities in B cell receptor (BCR) signaling may also provide a possible explanation. Prior work by $\mathrm{Ng}$ et al. suggests that absence of Btk contributes to a mechanism of inappropriate continuous receptor editing by $\mathrm{B}$ cells (whereas receptor recombination is typically downregulated by signaling through the BCR) [24]. This enables B cells, including autoreactive B cells, to overcome Btk-responsible maturation defects and escape to the periphery [24]. Because B cells rely on survival signaling through a Btk mechanism, it is theorized that those cells with self-reactive receptors are able to survive in the periphery due to signaling by self-antigens [24].

COMMON VARIABLE IMMUNE DEFICIENCY—Common variable immunodeficiency is a heterogeneous disease of unclear inheritance and pathogenesis that causes loss of memory B cells and isotype-switched B cells leading to hypogammaglobulinemia as well as varied $\mathrm{T}$ cell phenotypes. Clinical manifestations largely include sinopulmonary infection, lymphoid or granulomatous proliferative disorders, gastrointestinal disease, and autoimmunity [10, 13]. CVID most commonly occurs sporadically in $80-90 \%$ of patients in their 2nd or 3rd decade of life [26]. There have been five genetic defects described, which include the following: transmembrane activator and calcium-modulator and cyclophilin ligand interactor (TACI), inducible costimulator (ICOS), B-cell activating factor (BAFF) receptor, CD19 and mutS homolog 5 (MSH5) $[8,10]$, but the majority of patients have an unknown molecular cause. TACI mutation is found in $10 \%$ of patients with CVID and can be inherited in an autosomal dominant manner [9]. This particular molecular group has a higher incidence of autoimmunity associated with it, particularly autoimmune cytopenias, albeit mutation of TACI receptor has also been found in normal controls [21].

Autoimmune manifestations are found in roughly $25 \%$ of all CVID patients and may be the earliest presentation of the disorder [27]. Autoimmune disorders include cytopenias (11\%), idiopathic thrombocytopenia (ITP)/AHA (5-8\%), pernicious anemia (1-9\%), thyroiditis, rheumatoid arthritis (RA) (1-10\%), IBD or villous atrophy disease (6-10\%), hepatitis, PBC, alopecia, dermatomyositis, and vitiligo [10, 13, 20, 25, 26, 28-30]. Other lymphoproliferative diseases are also seen, including interstitial pneumonia, sarcoid-like granulomas (5-20\%), and lymphoma [11, 28, 29].

Although the mechanisms underlying CVID and autoimmune susceptibility are not well defined, several theories have been proposed. These include B cell abnormalities in peripheral tolerance, signaling and maturation, as well as loss of $\mathrm{T}$ regulatory cells (Tregs). It is suggested that autoreactive B cell removal in CVID depends on appropriate BCR signaling and may also be affected by impaired calcium signaling as seen in some B cell populations in CVID [31-33]. Additionally, immune complexes and FcyRIIB signaling provide negative feedback on B cells through inhibitory receptors; some hypothesize that decreased levels of IgG may contribute to failure of this regulatory effect [33]. In support of this, mouse models with a lupus-like disease have a loss of function of FcyRIIB and chronic B cell activation with increased germinal center and plasma cell accumulation, suggesting a loss of peripheral tolerance $[32,33]$. Alternatively, altered B cell survival factors, as seen in 
CVID with B cell activating factor (BAFF) and a proliferation-inducing ligand (APRIL), may contribute to autoreactive B cell survival $[31,33]$.

CVID patients may also have abnormalities in B cell sub-population maturation (such as memory and class-switched memory B cells) [21, 31]. An increased population of immature $\mathrm{B}$ cells has been associated with granulomatous and autoimmune disease, such as cytopenias [27, 34]. This expanded CD21low subset has been shown to express a polyclonal and unmutated BCR repertoire and has a higher percentage of auto-reactive clones that produce autoantibodies against cytoplasmic structures [34]. The full elicitation of these mechanisms is still under investigation.

$\mathrm{T}$ cell abnormalities in proliferation, differentiation, and signaling are also found in patients with CVID and can directly affect humoral immune function through effects on B cell maturation, survival, and function [28]. Additionally, Tregs are decreased in patients with CVID, and this may be due to changes in the strength of TCR signaling that are important in the development of tolerogenicity [20, 27, 33]. Cytokine aberrancies, such as IL-2 and 12 deficiencies, may also contribute to lack of elimination of autoreactive cells or other suppressive pathways such as seen in dendritic cell influence on Tregs [27].

IMMUNODYSREGULATION, POLYENDOCRINOPATHY, ENTEROPATHY, XLINKED-IPEX results from a defect in the transcription factor FoxP3, which is crucial to the development of $T$ regulatory cells (Tregs) $[3,10,12,23,35]$. The disease typically manifests as diabetes, thyroiditis, severe watery diarrhea, failure to thrive, and eczema within the first few months of life $[12,36]$. In most cases, IPEX is a fatal disease and causes death before the age of two [10]. Other autoimmune manifestations that occur in $100 \%$ of patients include enteritis, urticaria, T1DM, AHA, cytopenias, membranous nephropathy, pemphigoid nodularis, psoriasiform dermatitis, and alopecia $[4,8,10,12,20,23,35,36]$. Less commonly, patients may develop vasculitis, sarcoidosis, hepatitis, vitiligo, and adrenal failure [36].

Tregs form long-lasting synapses with antigen-presenting dendritic cells to inhibit effector $\mathrm{T}$ cell responses and induce peripheral tolerance [4]. Mutation of FoxP3 leads to reduced number and diversity of regulatory cells, thus allowing normally "hidden" antigenic epitopes to remain exposed. The resulting stimulation and proliferation of CD4+ T cells leads to an overproduction of inflammatory cytokines and the potential production of autoantibodies [4, 37]. Additionally, Tregs depend on IL-2 signaling for survival; hence, defects in IL-2 signaling (i.e. STAT5b, IL-2 receptor defects, IL-2 defects) can lead to an IPEX-like syndrome despite having normal FoxP3 expression [16, 20, 38]. Interestingly, in mice with FoxP3 deficiency, blocking two TNF receptors (OX40 and CD30) responsible for CD4+ memory and effector cell generation is protective against the development of autoimmune disease [39].

\section{DEFECTS IN CELLULAR GROWTH AND SURVIVAL}

\section{A. IMMUNOPROLIFERATION}

In some PID, the development of T and B cells is abnormal, resulting in a pronounced deficiency whereby the available lymphocyte repertoire clonally expands to fill the available niche. Resultant autoreactive $\mathrm{T}$ and $\mathrm{B}$ cells escape and proliferate, and autoimmunity may result. This concept is best observed in Omenn syndrome (OS) and DiGeorge syndrome, which result from RAG1/2 and defective thymic development, respectively.

OMEN SYNDROME-Omenn syndrome (OS) is an autosomal recessive condition primarily caused by mutations in RAG1 or RAG2, two enzymes necessary for T cell and B 
cell receptor arrangement $[16,40]$. Failure of this process arrests T cell and B cell development and results in decreased numbers of each [41]. Some patients with OS also have decreased AIRE gene expression in the thymus and mononuclear cells, similar to that seen in APECED [2, 4, 42]. Autoimmune manifestations occur in $100 \%$ of cases and are primarily directed against epithelium and the gastrointestinal tract; these include dermatitis, alopecia, hepatitis, and diarrhea $[9,20]$. Autoimmunity is felt to result from clonal expansion that includes autoreactive $\mathrm{T}$ cells directed against a variety of autoantigens as well as defective Treg production due to RAG defects [41].

DIGEORGE SYNDROME-DiGeorge syndrome is an autosomal dominant condition resulting in partial or complete failure of $\mathrm{T}$ cell development due to lack of or defective thymus development (as well as parathyroid and conotruncal regions of the heart), secondary to chromosome 22q11 deletion in the majority of cases $[16,40]$. While phenotype varies significantly, many of these patients also have facial dysmorphisms, neuropsychiatric disorders, cardiac abnormalities, parathyroid abnormalities, and deafness [43, 44]. Autoimmune manifestations are said to occur in 5-30\% of patients and are reported to have included cytopenias, AHA, hepatitis, IBD, arthritis, psoriasis, vitiligo, and thyroiditis [8, 20, $25,45-48]$.

It is presumed that rapid proliferation, such as in the case of OS or DiGeorge syndrome, may also lead to the clonal expansion of autoreactive cells by favoring the selection of $\mathrm{T}$ cells with a high affinity to self, as has been observed in T1DM, RA, Sjogren's syndrome, and systemic lupus erythematosus (SLE) [49]. While lymphopenia may be the stimulus required for proliferation, it has been suggested that several other "hits" may be required as a predisposition to this phenomena; these theories include increased numbers of autoreactive $\mathrm{T}$ cells due to failure of central or peripheral tolerance, restricted Treg diversity, impaired $\mathrm{T}$ cell survival, and the presentation of self-antigens due to tissue or cellular damage [16, 37, 49]. Additionally, some argue that exogenous microbial exposure may play a role in this mechanism, since lymphopenia in and of itself is not sufficient for homeostatic proliferation or an increased propensity toward autoimmune disease [37].

\section{B. APOPTOSIS}

Cell death is important in the control of immune response. When cell death does not occur as it should, inappropriate immune activation and persistence of aberrant cellular responses may occur that could predispose to autoimmunity. This concept is best observed in the primary immunodeficiency autoimmune lymphoproliferative syndrome (ALPS) which results from mutation of proteins involved in programmed cell death signaling: Fas [CD95, ALPS 0 (autosomal recessive) and ALPS 1a (autosomal dominant)], Fas ligand (ALPS 1b), caspase 10 (ALPS IIa), or caspase 8 (ALPS IIb) [4, 10, 40, 50, 51]. There is one additional ALPS subset (ALPS III) for which there is as yet no known molecular defect [40].

AUTOIMMUNE LYMPHOPROLIFERATIVE SYNDROME)-ALPS results in a clinical scenario of lymphocytosis and hypergammaglobulinemia, together with splenomegaly and lymphadenopathy, due to mutations in programmed cell death proteins. Diagnosis is typically made in early childhood [10,12]. Autoimmune manifestations have been reported in approximately 50-80\% of individuals and most often include cytopenias (ITP and neutropenia) and AHA but also glomerulonephritis, optic neuritis, Guillain-Barre syndrome, arthritis, cutaneous vasculitis, $\mathrm{PBC}$, hepatitis, blistering dermatosis, and acquired factor VIII deficiency $[8,10,20,51]$.

Fas is a member of the tumor necrosis factor (TNF) receptor family and is found on a diversified array of both immune and non-immune cell lines as is its ligand, Fas ligand 
(FasL). It is upregulated upon lymphocyte activation and induces programmed cell death several days later through the recruitment of death domains with subsequent signaling through caspase and the Ras pathway [50, 51]. In ALPS, autoreactive lymphocytes proliferate freely, and an increased burden of cellular debris may contribute to the development of more and diverse autoreactive cells. In these patients, unchecked autoreactive B cell stimulation leads to persistent autoantibody production, and autoreactive $\mathrm{T}$ cells persist due to impaired $\mathrm{T}$ cell receptor mediated apoptosis $[8,49,50]$. Additionally, when an increased burden of autoantigen and cellular debris overwhelms macrophage clearance, exposed self-antigens become available to antigen-presenting cells, allowing for the development of additional autoreactive $\mathrm{T}$ cells $[1,4,52]$. In a similar manner, toll-like receptors (TLR) 3 and 9 of the innate immune system are also able to recognize and crosspresent DNA released from dying cells and may contribute to further expansion of autoreactive T cells [4].

COMBINED IMMUNE DEFICIENCY SYNDROMES-ALPS is a prototypic example of dysregulated apoptosis with predisposition to autoimmunity; however, one newly described PID also points to defects in cell survival and function that are outside the Fas pathway. Lopez-Herrera et al. identified hypogammaglobulinemia (of at least two classes- $\operatorname{IgA}$, IgG, IgM), autoimmunity [ITP, AHA, thyroiditis, myasthenia gravis (MG), atrophic gastritis], and IBD or enteropathy in four patients with homozygous mutation in lipopolysaccharide responsive beige-like anchor protein (LRBA) [53]. Interestingly, LRBA is found most predominantly in immune cells and contains motifs important in compartmentalization of signaling enzymes, signal transduction, vesicular trafficking, cytoskeleton assembly, apoptosis, cell cycle, and transcription regulation [53]. B cells from this group of patients fail to proliferate, differentiate into plasma cells, and class-switch [53]. These cells also accumulate an abnormal number of organelles, suggesting a defect in autophagy, and EBVinfected cells in these patients have increased susceptibility to apoptosis [53].

Interestingly, immune dysfunction in Chediak-Higashi syndrome (CHS) is hypothesized to result from impaired vesicular trafficking due to mutations involving LRBA function. CHS is an immunodeficiency syndrome that manifests as recurrent infection, progressive neurological deterioration, hypopigmentation, and the accumulation of giant intracellular vesicles [54]. LRBA shares similarity to CHS1/beige and A kinase anchor genes already shown to be involved in CHS and to negatively impact the function of natural killer (NK) cells, cytotoxic T cells and granulocytes [54]. Although the autoimmune diseases of LRBA mutations are not overlapping with CHS, the neutropenia of CHS has been suggested to potentially originate from autoimmune origins [55].

\section{DEFECTS OF SIGNALING PATHWAYS}

Cells of the immune system are dependent on numerous intracellular signaling pathways leading to appropriate immune function. When signaling pathways are abnormal, impaired cytokine production, immune activation, and cellular migration can lead to broad defects in $\mathrm{T}, \mathrm{B}$, and innate immune cell function. This concept is best observed in Wiskott-Aldrich syndrome (WAS), which results from an X-linked defect in Wiskott-Aldrich syndrome protein (WASp) causing impaired actin polymerization and cytoskeletal function of hematopoietic cells $[1,16]$.

\section{WISKOTT-ALDRICH SYNDROME}

WAS is clinically characterized by thrombocytopenia with bleeding, eczema, and recurrent sinopulmonary infections and occurs in 1-10 per million males [1, 44, 52, 56]. Autoimmune manifestations occur in $40-72 \%$ of patients and have included cytopenias (typically 
neutropenia), AHA, vasculitis, arthritis, angioedema, myositis, dermatomyositis, IBD, hepatitis, uveitis, and renal disease $[1,10,13,20,25,40,52,56]$. As many as $25 \%$ of WAS patients have multiple autoimmune diseases [1], and autoimmunity is associated with an increased risk of malignancy and mortality [52]. Interestingly, the more mild X-linked form of WAS has been most predominantly associated with autoimmune manifestations $[1,56]$.

T cells are affected by defective WASp. Because of impaired cytoskeleton function, patients with WAS have unstable immune synapses and suboptimal signaling through the T cell receptor (TCR) [4]. TCR-activation is dependent on the recruitment of nuclear factor of activated T cell (NFAT 1 and 2), both of which are reduced in WAS patients and correlate with diminished Th1 cytokine production [52]. T effector cells, normally eliminated after prolonged exposure to antigen (including self-antigen), fail to undergo the same degree of TCR-mediated apoptosis when lacking WASp [52], and Treg numbers and function are deficient likely due to defective cytokine release and impaired intracellular granule movement $[1,9,16,20,23,52,56-59]$.

B cell receptor (BCR) interactions and signaling are also impaired; this is due to failure of WASp interaction with tyrosine kinases, including Bruton's tyrosine kinase (Btk) [52, 59, 60]. Consequently, there are fewer germinal centers and decreased migration, proliferation, and survival of B cells [52, 56, 59]. WASp-deficient B cells also express fewer complement receptors (CD21), contributing to an inability to effectively capture and present antigens and a possible breakdown in peripheral tolerance [52].

Likewise, innate cell dysfunction also occurs in WAS patients [23, 56, 58]. For example, natural killer (NK) cells demonstrate defective cytolytic function, and, in mouse models, restoration of a normal natural killer T cell (NKT) population can prevent the onset of diabetes, suggesting a role in autoimmunity [1]. Macrophage function is also impaired and hypothesized to contribute to autoimmunity through an inability to clear apoptotic debris $[1$, 52]. Additionally, dendritic cells lacking WASp may contribute to autoimmunity due to a proinflammatory cascade that results from an inability to negatively regulate type one interferon production after stimulation of toll-like receptor 9 [61]. These types of impairments are likely contributing factors in the development of autoimmunity in WAS.

\section{DEFECTS OF IMMUNE MEDIATED CLEARANCE}

An additional theory of autoimmune pathogenesis in PID is that of defects in antigen clearance. In some instances, it has been proposed to be secondary to molecular mimicry where increased susceptibility and recurrent infection ultimately results in tissue destruction, cellular activation, and impaired debris clearance that may lead to targeting of self-proteins and breaks in tolerance. This concept is one of the potential mechanisms theorized to be relevant in selective IgA deficiency and deficiencies of the classical complement pathway.

\section{SELECTIVE IgA DEFICIENCY}

Selective IgA deficiency (IgAD) is the most common form of PID, occurring in approximately 1 in 500 Caucasians [25, 44, 62], and is diagnosed when serum IgA levels are $\$ 7 \mathrm{mg} / \mathrm{dL}$ in a patient greater than 4 years old with normal levels of IgG and IgM [62, 63]. IgAD is associated with both organ-specific and systemic autoimmune manifestations in 7$36 \%$ of patients [62]. These have included SLE (1-5\%), RA (2-4\%), and celiac disease $(10-20 \%)$ as well as sporadic cases of urticaria, thyroiditis, AHA, ITP, T1DM, MG, psoriasis, vitiligo, and pemphigus $[8,11,13,20,25,62,63]$. The most common hematological autoimmune manifestation is ITP occurring in 1 in 200 patients [62]. 
The genetic defect responsible for selective IgA deficiency has not been elicited. However, as circulatory B cells in IgAD express surface IgA but lack secretory IgA, it has been suggested that a transcription or class switch defect may contribute to the clinical phenotype [8]. Additionally, a mutation in transmembrane activator and calcium-modulator and cyclophilin ligand interactor (TACI), a member of the tumor necrosis receptor family which mediates isotype switching, was identified in one patient with selective IgAD [62]; TACI mutation is also associated with CVID (see above). Genetic susceptibility linking IgA deficiency and CVID with autoimmunity is suggested by a shared susceptibility locus on chromosome 6 (DQ-DR) along with SLE, T1DM, thyroiditis, MG, and celiac disease [8, 63]. A genome-wide association study has revealed two additional non-HLA gene signals, IFIHI (MDA-5) (also significantly associated with T1DM and SLE) and CLEC16A (also significantly associated with T1DM and multiple sclerosis) [64].

At this time, autoimmune disease in IgAD is hypothesized to result from the lack of clearance of antigens from mucosal surfaces with resultant immune complex deposition. This leads to tissue damage and ongoing inflammation with subsequent exposure of autoantigens and breakdown in peripheral tolerance $[1,13]$. It has been proposed that these reactive lymphocytes may proliferate when responding to autoantigens presented by dendritic cells [1]. A second theory is that the lack of clearance of intraluminal antigens (from diet or pathogens) with molecular mimicry to normal tissue or the exposure to superantigens leads to a breakdown of peripheral tolerance [8, 63]. A third hypothesis, not involving clearance defects but in line with CVID, is the lack of inhibitory signaling through constitutively-expressed FcaR1 in patients with IgA deficiency [62].

\section{COMPLEMENT DEFICIENCIES}

Classical complement deficiencies are inherited in an autosomal recessive fashion and lead to an increased risk of the development of systemic lupus erythematosus (SLE) depending on the component affected [40]. SLE is found in $90 \%$ of patients with C1q deficiency, $75 \%$ of patients with $\mathrm{C} 4$ complete deficiency, $50-65 \%$ of patients with $\mathrm{C} 1 \mathrm{r} / \mathrm{s}$ deficiencies, and $10-30 \%$ of patients with homozygous $\mathrm{C} 2$ deficiency [10, 20, 25]. Of these complement component deficiencies, homozygous $\mathrm{C} 2$ deficiency is the most common, occurring in 1 in 10,000 to 1 in 30,000 Caucasians, and is caused by a premature stop codon [10]. Neither heterozygous $\mathrm{C} 2$ deficiency nor homozygous $\mathrm{C} 4 \mathrm{~B}$ deficiency has been associated with increased autoimmunity [10].

Each of these components is important in the clearance of immune complexes and other apoptotic bodies. If this debris is not cleared, there is a hypothesized increased exposure of intranuclear antigens leading to the formation of antinuclear antibodies (ANA) [8].

Alternatively, some argue that ANA are the result of tissue damage that occurs from immune complex deposition (due to lack of clearance) as has been shown in burn patients in which ANA are produced [8].

\section{DEFECTS OF INNATE CELLULAR MECHANISMS}

Innate immune cells contribute to the normal functioning of the adaptive immune system, and defects may contribute to disease pathogenesis in autoimmunity. In these cases, autoimmunity is hypothesized to result from inappropriate cellular activation and inflammation and the failure of development of peripheral tolerance mechanisms. This concept is best observed in chronic granulomatous disease (CGD) resulting from defective granulocyte bactericidal activity. 


\section{CHRONIC GRANULOMATOUS DISEASE}

CGD occurs in 1 in 250,000 persons and is the result of one of four defects in the nicotinamide adenine dinucleotide phosphate (NADPH) oxidase complex $[65,66]$. This results in an inability to transfer an electron from NADPH to oxygen and produce reactive oxygen species (ROS) superoxide [66, 67]. This functional phagocytic disorder presents with recurrent abscesses, osteomyelitis, pneumonia, lymphadenitis, and granulomatous formation [25]. There is one X-linked defect involving the component gp91phox (65\% of cases) and three autosomal recessive defects involving the components p47phox (25\%), p67phox (5\%), and p22phox (5\%) [66]. Those patients with X-linked disorders tend to have more severe presentations, more severe infections, and earlier death [66].

Autoimmune manifestations most commonly include CGD-associated colitis (histologically similar to Crohn's disease) and discoid lupus [25, 68]. Other manifestations include sarcoidosis, ITP, RA, juvenile arthritis, and celiac disease [25, 66, 68, 69]. Unaffected carriers of the disease have also been reported to have an increased incidence of discoid lupus $[11,65]$. Preliminary molecular work suggests polymorphisms of myeloperoxidase (MPO), Fc $\gamma$ RIIa, and Fc $\gamma$ RIIIb are associated with GI inflammation and granuloma formation, while variant alleles of mannose binding lectin (MBL) and $\mathrm{FC} \gamma \mathrm{RIIa}$ are associated more with rheumatological manifestations [69].

Autoimmune manifestations are hypothesized to result from impaired Treg production, persistent cellular activation with loss of peripheral tolerance, and poor debris clearance causing increased exposure to autoantigens. In vitro evidence has shown that ROS production by macrophages leads to the development of Tregs [5]. Therefore, a ROSdeficient state may lead to diminished Treg production and loss of peripheral tolerance [5, $67,68]$. Persistent cellular activation and inflammation may additionally occur due to impaired ROS-driven apoptosis, silencing of inflammatory mediators (such as leukotrienes), granulocyte apoptosis, or degradation of material $[5,67,68]$. Similarly, altered innate pathogen recognition and chemotactic receptor expression may also affect cellular activation and function [67]. Additionally, a defect in the kynuenine pathway (which favors immune tolerance) may play a role as kynuenine replacement has been shown to reverse inflammation in a mouse model of CGD [67]. Finally, poor debris removal (due to decreased expression of phosphatidyl serine) may cause increased exposure to autoantigens $[65,69]$. These postulated mechanisms are still being studied in this PID.

\section{CONCLUSIONS}

The understanding of autoimmunity in PID has made great strides over the past years, due in large part to gains in knowledge about PID mechanisms. As such, the above-described PIDs highlight currently accepted theories of autoimmunity in these conditions (defects of tolerance, apoptosis, immunoproliferation, signaling pathways, immune-mediated clearance, and innate cellular mechanisms). Ongoing and future exploration are sure to push this frontier forward, improving our insight into the pathogenesis of autoimmunity, revealing new arenas for study, and, ultimately, providing targets for treatment for patients who suffer the consequences of an immune system gone awry.

\section{Abbreviations}

APECED Autoimmune polyendocrinopathy candidiasis ectodermal dysplasia

HIgM Hyper IgM syndrome

XLA

$\mathrm{X}$-linked agammaglobulinemia 


$\begin{array}{ll}\text { CVID } & \text { Common variable immunodeficiency } \\ \text { IPEX } & \text { Immunodysregulation polyendocrinopathy enteropathy x-linked } \\ \text { OS } & \text { Omenn syndrome } \\ \text { ALPS } & \text { DiGeorge syndrome; Autoimmune lymphoproliferative syndrome } \\ \text { WAS } & \text { Wiskott Aldrich syndrome } \\ \text { IgAD } & \text { IgA deficiency } \\ \text { CGD } & \text { Complement deficiency; Chronic granulomatous disease }\end{array}$

\section{REFERENCES}

1. Schurman SH, Candotti F. Autoimmunity in Wiskott-Aldrich syndrome. Curr Opin Rheumatol. 2003; 15(4):446-453. [PubMed: 12819473]

2. Marrella V, Poliani P, Fontana E, et al. Anti-CD3epsilon mAb improves thymic architecture and prevents autoimmune manifestations in a mouse model of Omenn syndrome: therapeutic implications. Blood. 2012; 120(5):1005-1014. [PubMed: 22723555]

3. Michels AW, Gottlieb PA. Autoimmune polyglandular syndromes. Nat Rev Endocrinol. 2010; 6(5): 270-277. [PubMed: 20309000]

4. Westerberg LS, Klein C, Snapper SB. Breakdown of T cell tolerance and autoimmunity in primary immunodeficiency--lessons learned from monogenic disorders in mice and men. Curr Opin Immunol. 2008; 20(6):646-654. [PubMed: 18955138]

5. Kraaij MD, Savage ND, van der Kooij SW, et al. Induction of regulatory T cells by macrophages is dependent on production of reactive oxygen species. Proc Natl Acad Sci U S A. 2010; 107(41): 17686-17691. [PubMed: 20861446]

6. Ramsey C, Wingvist O, Puhakka L, et al. Aire deficient mice develop multiple features of APECED phenotype and show altered immune response. Hum Mol Genet. 2002; 11(4):397-409. [PubMed: 11854172]

7. Rizzi M, Ferrera F, Filaci G, et al. isruption of immunological tolerance: role of AIRE gene in autoimmunity. Autoimmun Rev. 2006; (2):145-147. [PubMed: 16431348]

8. Arason GJ, Jorgensen GH, Ludviksson BR. Primary immunodeficiency and autoimmunity: lessons from human diseases. Scand J Immunol. 2010; 71(5):317-328. [PubMed: 20500682] This paper discusses autoimmunity in primary immunodeficiencies as monogenic and polygenic disorders. It also contrasts this discussion with other primary immunodeficiencies not strongly associated with autoimmunity.

9. Goyal R, Bulua AC, Nikolov NP, et al. Rheumatologic and autoimmune manifestations of primary immunodeficiency disorders. Curr Opin Rheumatol. 2009; 21(1):78-84. [PubMed: 19077721]

10. Bussone G, Mouthon L. Autoimmune manifestations in primary immune deficiencies. Autoimmun Rev. 2009; 8(4):332-336. [PubMed: 19028607]

11. Grammatikos AP, Tsokos GC. Immunodeficiency and autoimmunity: lessons from systemic lupus erythematosus. Trends Mol Med. 2012; 18(2):101-108. [PubMed: 22177735]

12. Lehman HK, Ballow M. Immune deficiency disorders with autoimmunity and abnormalities in immune regulation-monogenic autoimmune diseases. Clin Rev Allergy Immunol. 2008; 34(2): 141-145. [PubMed: 18330725]

13. Patiroglu T, Gungor HE, Unal E. Autoimmune diseases detected in children with primary immunodeficiency diseases: results from a reference centre at middle anatolia. Acta Microbiol Immunol Hung. 2012; 59(3):343-353. [PubMed: 22982638]

14. Su MA, Davini D, Cheng P, et al. Defective autoimmune regulator-dependent central tolerance to myelin protein zero is linked to autoimmune peripheral neuropathy. J Immunol. 2012; 188(10): 4906-4912. [PubMed: 22490868] 
15. Teh CE, Daley SR, Enders A, et al. T-cell regulation by casitas B-lineage lymphoma (Cblb) is a critical failsafe against autoimmune disease due to autoimmune regulator (Aire) deficiency. Proc Natl Acad Sci U S A. 2010; 107(33):14709-14714. [PubMed: 20668237]

16. Atkinson TP. Immune deficiency and autoimmunity. Curr Opin Rheumatol. 2012; 24(5):515-521. [PubMed: 22820513] This paper discusses the new concept of insufficient $\mathrm{T}$ cell regulatory function in the setting of aberrant effector T cell development.

17. Jesus AA, Duarte AJ, Oliveira JB. Autoimmunity in hyper-IgM syndrome. J Clin Immunol. 2008; 28(Suppl 1):S62-S66. [PubMed: 18246414]

18. Quartier P, Bustamante J, Sanal O, et al. Clinical, immunologic and genetic analysis of 29 patients with autosomal recessive hyper-IgM syndrome due to Activation-Induced Cytidine Deaminase deficiency. Clin Immunol. 2004; 110(1):22-29. [PubMed: 14962793]

19. Winkelstein JA, Marino MC, Ochs, et al. The X-linked hyper-IgM syndrome: clinical and immunologic features of 79 patients. Medicine (Baltimore). 2003; 82(6):373-384. [PubMed: 14663287]

20. Coutinho A, Carneiro-Sampaio M. Primary immunodeficiencies unravel critical aspects of the pathophysiology of autoimmunity and of the genetics of autoimmune disease. J Clin Immunol. 2008; 28(Suppl 1):S4-S10. [PubMed: 18293069]

21. Cunningham-Rundles C. Autoimmunity in primary immune deficiency: taking lessons from our patients. Clin Exp Immunol. 2011; 164(Suppl 2):6-11. [PubMed: 21466546] This paper succinctly summarizes current theories of autoimmunity in primary immunodeficiencies with some focus on B cell defects and CVID.

22. Davies EG, Thrasher AJ. Update on the hyper immunoglobulin M syndromes. Br J Haematol. 2010; 149(2):167-180. [PubMed: 20180797]

23. Pessach IM, Notarangelo LD. X-linked primary immunodeficiencies as a bridge to better understanding X-chromosome related autoimmunity. J Autoimmun. 2009; 33(1):17-24. [PubMed: 19361956]

24. Ng YS, Wardemann H, Chelnis J, et al. Bruton's tyrosine kinase is essential for human B cell tolerance. J Exp Med. 2004; 200(7):927-934. [PubMed: 15466623]

25. Etzioni A. Immune deficiency and autoimmunity. Autoimmun Rev. 2003; 2(6):364-369. [PubMed: 14550878]

26. Brandt D, Gershwin ME. Common variable immune deficiency and autoimmunity. Autoimmun Rev. 2006; 5(7):465-470. [PubMed: 16920573]

27. Lopes-da-Silva S, Rizzo LV. Autoimmunity in common variable immunodeficiency. J Clin Immunol. 2008; 28(Suppl 1):S46-S55. [PubMed: 18443901]

28. Agarwal S, Cunningham-Rundles C. Autoimmunity in common variable immunodeficiency. Curr Allergy Asthma Rep. 2009; 9(5):347-352. [PubMed: 19671377]

29. Giannouli S, Anagnostou D, Soliotis F, et al. Autoimmune manifestations in common variable immunodeficiency. Clin Rheumatol. 2004; 23(5):449-452. [PubMed: 15278751]

30. Knight AK, Cunningham-Rundles C. Inflammatory and autoimmune complications of common variable immune deficiency. Autoimmun Rev. 2006; 5(2):156-159. [PubMed: 16431351]

31. Cunningham-Rundles C. Autoimmune manifestations in common variable immunodeficiency. $\mathrm{J}$ Clin Immunol. 2008; 28(Suppl 1):S42-S45. [PubMed: 18322785]

32. McGaha TL, Karlsson MC, Ravetch JV. FcgammaRIIB deficiency leads to autoimmunity and a defective response to apoptosis in Mrl-MpJ mice. J Immunol. 2008; 180(8):5670-5679. [PubMed: 18390752]

33. Warnatz K, Voll RE. Pathogenesis of autoimmunity in common variable immunodeficiency. Front Immunol. 2012; 3:210. [PubMed: 22826712]

34. Boileau J, Mouillot G, Gerard L, et al. Autoimmunity in common variable immunodeficiency: correlation with lymphocyte phenotype in the French DEFI study. J Autoimmun. 2011; 36(1):2532. [PubMed: 21075598]

35. Bacchetta R, Passerini L, Gambineri E, et al. Defective regulatory and effector T cell functions in patients with FOXP3 mutations. J Clin Invest. 2006; 116(6):1713-1722. [PubMed: 16741580] 
36. Moraes-Vasconcelos D, Costa-Carvalho BT, Torgerson TR, et al. Primary immune deficiency disorders presenting as autoimmune diseases: IPEX and APECED. J Clin Immunol. 2008; 28(Suppl 1):S11-S19. [PubMed: 18264745]

37. Milner JD, Fasth A, Etzioni A. Autoimmunity in severe combined immunodeficiency (SCID): lessons from patients and experimental models. J Clin Immunol. 2008; 28(Suppl 1):S29-S33. [PubMed: 18193342]

38. Caudy A, Reddy ST, Chatila T, et al. CD25 deficiency causes an immune dysregulation, polyendocrinopathy, enteropathy, X-linked-like syndrome, and defective IL-10 expression from CD4 lymphocytes. J Allergy Clin Immunol. 2007; 119(2):482-487. [PubMed: 17196245]

39. Gaspal F, Withers D, Saini M, et al. Abrogation of CD30 and OX40 signals prevents autoimmune disease in FoxP3-deficient mice. J Exp Med. 2011; 208(8):1579-1584. [PubMed: 21788408]

40. Mackay IR, Leskovsek NV, Rose NR. The odd couple: a fresh look at autoimmunity and immunodeficiency. J Autoimmun. 2010; 35(3):199-205. [PubMed: 20817405] This paper highlights a cross-disciplinary summary discussion of the American Autoimmune Related Disease Association (AARDA) on autoimmunity and immunodeficiency.

41. Marrella V, Poliani PL, Sabacchi C, et al. Of Omenn and mice. Trends Immunol. 2008; 29(3):133140. [PubMed: 18255337]

42. Somech R, Simon AJ, Lev A, et al. Reduced central tolerance in Omenn syndrome leads to immature self-reactive oligoclonal T cells. J Allergy Clin Immunol. 2009; 124(4):793-800. [PubMed: 19767069]

43. Gennery AR. Immunological aspects of 22q11.2 deletion syndrome. Cell Mol Life Sci. 2012; 69(1):17-27. [PubMed: 21984609]

44. Turvey SE, Bonilla FA, Junker AK. Primary immunodeficiency diseases: a practical guide for clinicians. Postgrad Med J. 2009; 85(1010):660-666. [PubMed: 20075404]

45. Davies JK, Telfer P, Cavenagh JD, et al. Autoimmune cytopenias in the 22q11.2 deletion syndrome. Clin Lab Haematol. 2003; 25(3):195-197. [PubMed: 12755799]

46. Gennery AR, Barge D, O'Sullivan JJ, et al. Antibody deficiency and autoimmunity in 22q11.2 deletion syndrome. Arch Dis Child. 2002; 86(6):422-425. [PubMed: 12023174]

47. McLean-Tooke A, Barge D, Spickett GP, et al. Immunologic defects in 22q11.2 deletion syndrome. J Allergy Clin Immunol. 2008; 122(2):362-367. 367 e1-367 e4. [PubMed: 18485468]

48. Tison BE, Nicholas SK, Abramson SL, et al. Autoimmunity in a cohort of 130 pediatric patients with partial DiGeorge syndrome. J Allergy Clin Immunol. 2011; 128(5):1115-1117. e1-e3. [PubMed: 21835443]

49. Baccala R, Theofilopoulos AN. The new paradigm of T-cell homeostatic proliferation-induced autoimmunity. Trends Immunol. 2005; 26(1):5-8. [PubMed: 15629402]

50. Famularo G, Nucera E, Marcellini, et al. Fas/Fas ligand on the road: an apoptotic pathway common to AIDS, autoimmunity, lymphoproliferation and transplantation. Med Hypotheses. 1999; 53(1): 50-62. [PubMed: 10499826]

51. Ramenghi U, Bonissoni S, Migliaretti G, et al. Deficiency of the Fas apoptosis pathway without Fas gene mutations is a familial trait predisposing to development of autoimmune diseases and cancer. Blood. 2000; 95(10):3176-3182. [PubMed: 10807785]

52. Catucci M, Castiello MC, Pala F, et al. Autoimmunity in wiskott-Aldrich syndrome: an unsolved enigma. Front Immunol. 2012; 3:209. [PubMed: 22826711]

53. Lopez-Herrera G, Tampella G, Pan-Hammarstrom Q, et al. Deleterious mutations in LRBA are associated with a syndrome of immune deficiency and autoimmunity. Am J Hum Genet. 2012; 90(6):986-1001. [PubMed: 22608502]

54. Wang JW, Howson J, Haller E, et al. Identification of a novel lipopolysaccharide-inducible gene with key features of both A kinase anchor proteins and chs1/beige proteins. J Immunol. 2001; 166(7):4586-4595. [PubMed: 11254716]

55. Rezaei N, Moazzami K, Aghamohammadi A, et al. Neutropenia and primary immunodeficiency diseases. Int Rev Immunol. 2009; 28(5):335-66. [PubMed: 19811314]

56. Cleland SY, Siegel RM. Wiskott-Aldrich Syndrome at the nexus of autoimmune and primary immunodeficiency diseases. FEBS Lett. 2011; 585(23):3710-3714. [PubMed: 22036785] 
57. Marangoni F, Trifari S, Scaramuzza S, et al. WASP regulates suppressor activity of human and murine CD4(+)CD25(+)FOXP3(+) natural regulatory T cells. J Exp Med. 2007; 204(2):369-380. [PubMed: 17296785]

58. Snapper SB, Meelu P, Nguyen D, et al. WASP deficiency leads to global defects of directed leukocyte migration in vitro and in vivo. J Leukoc Biol. 2005; 77(6):993-998. [PubMed: 15774550]

59. Thrasher AJ, Burns SO. WASP: a key immunological multitasker. Nat Rev Immunol. 2010; 10(3): 182-192. [PubMed: 20182458]

60. Zhang L, Radigan L, Salzer U, et al. Transmembrane activator and calcium-modulating cyclophilin ligand interactor mutations in common variable immunodeficiency: clinical and immunologic outcomes in heterozygotes. J Allergy Clin Immunol. 2007; 120(5):1178-1185. [PubMed: 17983875]

61. Prete F, Catucci M, Labrada M, et al. Wiskott-Aldrich syndrome protein-mediated actin dynamics control type-I interferon production in plasmacytoid dendritic cells. J Exp Med. 2013; 210(2):355374. [PubMed: 23337808]

62. Jacob CM, Pastorino AC, Fahl K, et al. Autoimmunity in IgA deficiency: revisiting the role of IgA as a silent housekeeper. J Clin Immunol. 2008; 28(Suppl 1):S56-S61. [PubMed: 18202833]

63. Jorgensen GH, Thorsteinsdottir I, Gudmundsson S, et al. Familial aggregation of IgAD and autoimmunity. Clin Immunol. 2009; 131(2):233-239. [PubMed: 19167929]

64. Ferreira RC, Pan-Hammarstrom Q, Graham RR, et al. Association of IFIH1 and other autoimmunity risk alleles with selective IgA deficiency. Nat Genet. 2010; 42(9):777-780. [PubMed: 20694011]

65. Cale CM, Morton L, Goldblatt D. Cutaneous and other lupus-like symptoms in carriers of X-linked chronic granulomatous disease: incidence and autoimmune serology. Clin Exp Immunol. 2007; 148(1):79-84. [PubMed: 17286762]

66. Holland SM. Chronic granulomatous disease. Clin Rev Allergy Immunol. 2010; 38(1):3-10. [PubMed: 19504359]

67. Schappi MG, Jaquet V, Belli DC, et al. Hyperinflammation in chronic granulomatous disease and anti-inflammatory role of the phagocyte NADPH oxidase. Semin Immunopathol. 2008; 30(3):255271. [PubMed: 18509648]

68. De Ravin SS, Naumann N, Cowen EW, et al. Chronic granulomatous disease as a risk factor for autoimmune disease. J Allergy Clin Immunol. 2008; 122(6):1097-1103. [PubMed: 18823651]

69. Foster CB, Lehrnbecher T, Mol F, et al. Host defense molecule polymorphisms influence the risk for immune-mediated complications in chronic granulomatous disease. J Clin Invest. 1998; 102(12):2146-2155. [PubMed: 9854050] 
Table 1

Proposed mechanisms of autoimmunity in primary immunodeficiencies (PID).*

\begin{tabular}{|c|c|c|}
\hline PID & Genetic defect(s) & Proposed major mechanisms of autoimmunity \\
\hline \multicolumn{3}{|l|}{ Defects in tolerance } \\
\hline APECED & AIRE & $\begin{array}{l}\text { Failure of deletion of autoreactive } \mathrm{T} \text { cells; failure of anergy due to } \\
\text { loss of inhibitory signals }\end{array}$ \\
\hline HIgM & $\begin{array}{l}\text { CD } 40, \text { CD } 40 \mathrm{~L}, \text { AID, UDG, NEMO, } \\
\text { IkBa }\end{array}$ & $\begin{array}{l}\text { Failure of deletion of autoreactive B cells; loss of Treg development; } \\
\text { deranged T cell activation by innate system }\end{array}$ \\
\hline XLA & Btk & $\begin{array}{l}\text { Similar to HIgM (see above); continuous BCR editing with } \\
\text { peripheral survival advantage for autoreactive B cells }\end{array}$ \\
\hline CVID & $\begin{array}{l}\text { TACI, ICOS, BAFF-R, CD19, MSH5, } \\
\text { others unknown }\end{array}$ & $\begin{array}{l}\text { Proliferation of autoreactive B cells due to impaired BCR-TCR } \\
\text { interactions, loss of FcyRIIB inhibitory signaling, or altered BAFF/ } \\
\text { APRIL survival signaling; decreased Treg population due to cytokine } \\
\text { aberrancies or impaired TCR signaling }\end{array}$ \\
\hline IPEX & FoxP3 & $\begin{array}{l}\text { Decreased number and diversity of Treg cells; aberrant stimulation of } \\
\text { CD4+ T cells with subsequent production of autoantibodies }\end{array}$ \\
\hline \multicolumn{3}{|c|}{ Defects in cellular growth and survival } \\
\hline OS & RAG1/RAG2, +/-AIRE & $\begin{array}{l}\text { Autoreactive lymphocyte escape and proliferation; defective Treg } \\
\text { production }\end{array}$ \\
\hline DiGeorge Syndrome & Chromosome 22q11 deletion & $\begin{array}{l}\text { Failure of T cell development with autoreactive } \mathrm{T} \text { cell escape; } \\
\text { restricted Treg diversity; tissue or cellular damage with autoantigen } \\
\text { exposure and formation of autoantibodies and autoreactive T cells }\end{array}$ \\
\hline ALPS & Fas, FasL, caspase $8 \& 10$ & $\begin{array}{l}\text { Persistence of autoreactive B and T cells due to failure of apoptosis; } \\
\text { impaired debris clearance with autoantigen exposure and formation } \\
\text { of autoantibodies and autoreactive T cells }\end{array}$ \\
\hline \multicolumn{3}{|c|}{ Defects of signaling pathways } \\
\hline WAS & WASp & $\begin{array}{l}\text { Impairment of cytoskeletal movement/migration, signaling pathways } \\
\text { and cytokine production needed for inhibition of the inflammatory } \\
\text { cascade, Treg stimulation, apoptosis, and TCR/BCR interactions; } \\
\text { impaired debris clearance (see above) }\end{array}$ \\
\hline \multicolumn{3}{|c|}{ Defects of immune-mediated clearance } \\
\hline $\operatorname{Ig} \mathrm{AD}$ & Unknown, possible TACI & $\begin{array}{l}\text { Impaired debris clearance (see above ALPS); bystander activation; } \\
\text { impaired inhibitory signaling }\end{array}$ \\
\hline Complement Deficiencies & $\mathrm{C} 1 \mathrm{q}, \mathrm{C} 1 \mathrm{r} / \mathrm{s}, \mathrm{C} 4, \mathrm{C} 2$ & Impaired debris clearance and tissue damage (see above ALPS) \\
\hline \multicolumn{3}{|c|}{ Defects of innate cellular mechanisms } \\
\hline CGD & NADPH oxidase complex components & $\begin{array}{l}\text { Defective production of reactive oxygen species contributing to } \\
\text { impaired Treg production and T cell apoptosis; impaired debris } \\
\text { clearance (see above, ALPS) }\end{array}$ \\
\hline
\end{tabular}

$A P E C E D$ polyendocrinopathy, candidiasis, and ectodermal dystrophy; $A I D$ activation-induced cytidine deaminase, AIRE autoimmune regulator; $A L P S$ autoimmune lymphoproliferative syndrome; $A P R I L$ a proliferation inducing ligand; BAFF- $R \mathrm{~B}$ cell activating factor receptor; Btk Bruton's tyrosine kinase; $C D 4 O L$ CD40 ligand; $C G D$ chronic granulomatous disease; $C V I D$ common variable immunodeficiency; FasL Fas ligand; FoxP3 forkhead box protein P3; HigM hyper IgM syndrome; ICOS inducible costimulator; IgAD selective IgA deficiency; IPEX immunodysregulation, polyendocrinopathy, enteropathy, X-linked; $M S H 5$ mutS homolog 5; NADPH nicotinamide adenosine dinucleotide phosphate; NEMO kappa B essential modulator; $O S$ Omenn Syndrome; $T A C I$ transmembrane activator and calcium-modulator and cyclophilin ligand interactor; $U D G$ uracilDNA-glycosylase; $W A S(p)$ Wiskott-Aldrich Syndrome (protein); XLA X-linked agammaglobulinemia 